\title{
Téoros
}

Revue de recherche en tourisme

\section{Tendances et opportunités du tourisme au Moyen-Orient}

\section{Françoise Mommens}

Volume 25, numéro 2, été 2006

Désirs d’Orient : du passé vers l'avenir

URI : https://id.erudit.org/iderudit/1071067ar

DOI : https://doi.org/10.7202/1071067ar

Aller au sommaire du numéro

Éditeur(s)

Université du Québec à Montréal

ISSN

0712-8657 (imprimé)

1923-2705 (numérique)

Découvrir la revue

Citer cet article

Mommens, F. (2006). Tendances et opportunités du tourisme au Moyen-Orient. Téoros, 25(2), 47-52. https://doi.org/10.7202/1071067ar d'utilisation que vous pouvez consulter en ligne.

https://apropos.erudit.org/fr/usagers/politique-dutilisation/ 


\section{Tendances et opportunités du tourisme au Moyen-Orient}

\section{Françoise Mommens}

Pendant longtemps, la simple évocation du Moyen-Orient suggérait des images d'exotisme, des parfums d'eau de rose et d'églantier, bref une ambiance de conte des Mille et Une Nuits. Longtemps, nous l'avons idéalisé comme le berceau des premières civilisations et des premiers empires, à travers les légendes et les récits bibliques. Mais, depuis quelques années, ces images idylliques ont fait place aux visions de guerre, de polémiques et de déchirements. Les conflits économico-politiques qui hantent cette partie du globe nous donnent une impression de chaos, un relent de révolution (EIU, 2005 ; AME Info, 2005a).

Et pourtant, ces pays nous invitent, encore et toujours, à de grands voyages exploratoires, assurant que notre sécurité ne sera pas menacée. Comment se porte l'industrie du voyage au Moyen-Orient ? Qui en sont les principaux acteurs et quelle clientèle ceux-ci visent-ils ?

Le présent texte tente de comprendre pourquoi, malgré certaines idées reçues selon lesquelles on s'attend tout naturellement à ce que les touristes évitent les points chauds de l'actualité politique mondiale, le tourisme à destination du Moyen-Orient connaît une croissance plus rapide que celui de n'importe quelle autre région.

Sans insister sur les enjeux religieux et politiques, nous proposons un survol des perspectives et des volontés de développement du tourisme dans cette région, ainsi que des différentes stratégies de promotion utilisées dans quelques pays.

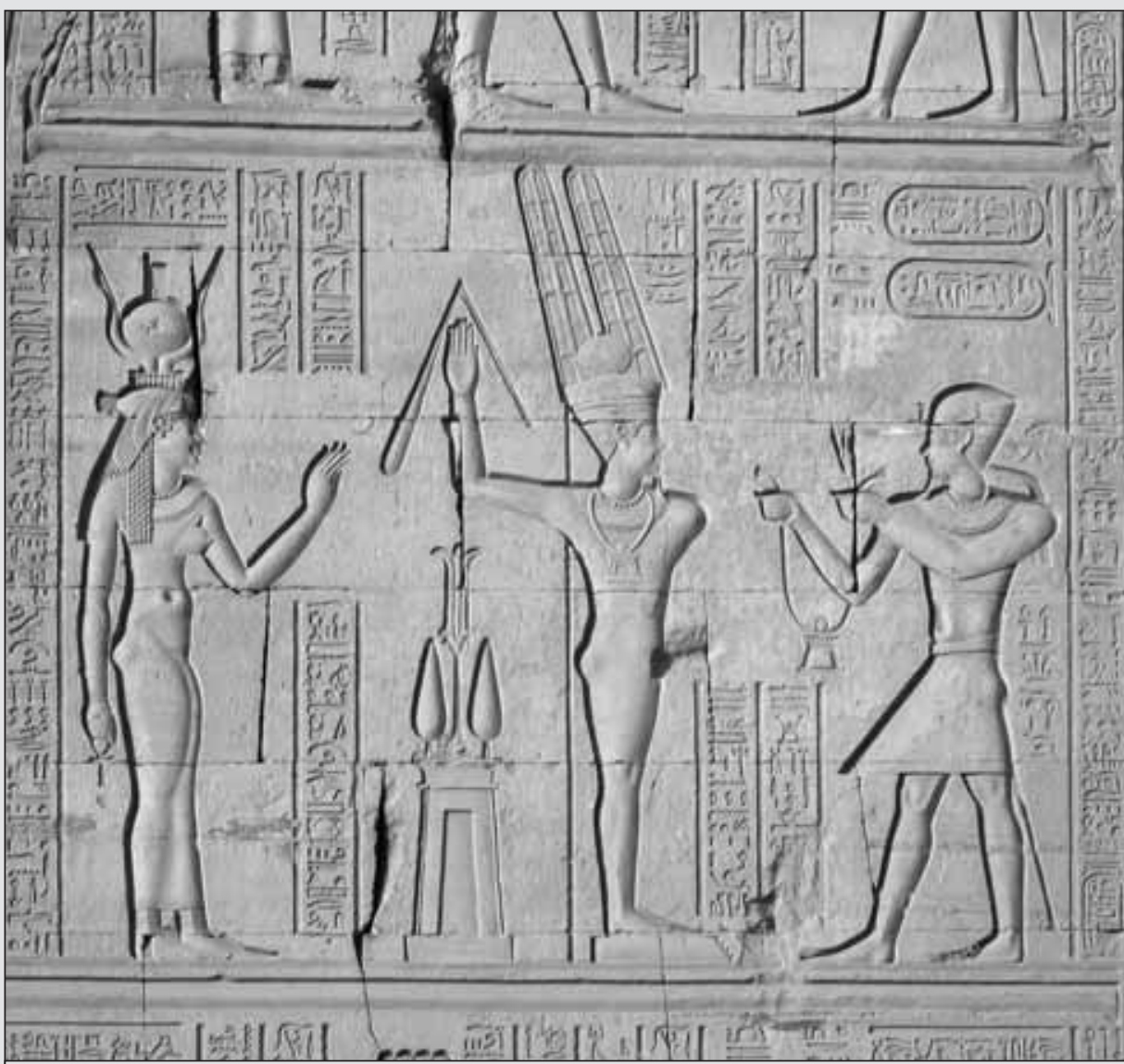

Hiéroglyphes égyptiens.

Photo: Michel Hassen/www.phototheque.net

\section{Le tourisme au Moyen-Orient: une inexorable progression}

Le Moyen-Orient est sans conteste, et depuis longtemps, un carrefour commercial décisif entre l'Europe, l'Asie et l'Afrique. Par sa position géographique, il est au cœur des affaires internationales, une région stratégique. En effet, il produit près de $35 \%$ du pétrole mondial et détient plus de $65 \%$ des réserves mondiales.
L'économie générale du Moyen-Orient se porte plutôt bien et, en raison d'une croissance constante, cette région témoigne d'une activité soutenue qui ne s'explique pas seulement par la récente embellie des cours du pétrole. En effet, d'importants programmes de restructuration et de remise à niveau sont à l'œuvre dans la plupart de ces pays (Younes, 2005). Le tableau 1 montre la croissance réelle du produit intérieur brut (PIB), de 1996 à 2005, dans les différents pays de la région. 
Tableau 1

\section{Croissance réelle du PIB, de 1996 à 2005 (en \%)}

$\begin{array}{lcccccccccc} & 1996 & 1997 & \mathbf{1 9 9 8} & \mathbf{1 9 9 9} & \mathbf{2 0 0 0} & \mathbf{2 0 0 1} & \mathbf{2 0 0 2} & \mathbf{2 0 0 3} & \mathbf{2 0 0 4} & \mathbf{2 0 0 5} \\ \begin{array}{lcccccccc}\text { Arabie } \\ \text { saoudite }\end{array} & 1,4 & 2,5 & (1,0) & 1,6 & 4,1 & 1,7 & 0,6 & 3,5 & 5,3 & 4,7 \\ \text { Bahrein } & 3,1 & 3,1 & 3,1 & 1,2 & 5,2 & 5,0 & 3,8 & 4,9 & 5,6 & 6,4 \\ \text { Égypte } & 5,1 & 5,9 & 5,0 & 3,1 & 3,9 & 2,5 & 3,0 & 1,8 & 2,7 & 3,8 \\ \text { Émirats } & 3,0 & 1,0 & (5,0) & (1,2) & 5,0 & 2,9 & 2,4 & 3,5 & 5,7 & 5,8 \\ \text { arabes unis } & & & & & & & & & \\ \text { Jordanie } & 0,8 & 2,7 & 2,2 & 1,5 & 2,5 & 2,8 & 3,6 & 3,1 & 6,4 & 6,0 \\ \text { Liban } & 4,0 & 3,5 & 3,5 & 4,0 & 0,0 & 0,8 & 0,9 & 2,0 & 4,0 & 4,5 \\ \text { Oman } & 3,5 & 3,5 & 2,1 & 1,5 & 4,7 & 5,0 & 2,2 & 1,1 & 1,2 & 1,4 \\ \text { Qatar } & 8,9 & 10,5 & 3,2 & 3,3 & 4,3 & 5,7 & 4,6 & 8,5 & 8,7 & 8,3 \\ \text { Syrie } & 5,5 & 4,1 & 3,4 & 4,0 & 1,5 & 1,6 & 2,2 & 0,9 & 2,2 & 1,9\end{array}$

Source : « Middle East Hotels Market: Outlook, Trends and Opportunities », HVS International, 2005, à partir des données de The Economist Intelligence Unit de février 2005.

Peu à peu, et à des vitesses différentes, ces pays se tournent vers l'industrie du tourisme afin de compenser une économie unique, difficile ou en déclin. Le Moyen-Orient est composé de pays dont la richesse est notoire et d'autres, plus pauvres, qui tentent de rivaliser d'imagination et de culture afin d'attirer à eux la manne touristique et ses revenus économiques. Dans la catégorie des plus riches et des plus connus, citons les Émirats arabes unis (EAU), l'Arabie saoudite et l'Égypte.

\section{Les Émirats arabes unis}

Ce pays dispose d'une prospérité étroitement liée à l'industrie du pétrole (8\% des réserves mondiales) et du gaz, qui représente plus de 30 \% du PIB. En 2005, les EAU sont le troisième producteur de pétrole dans le golfe Persique, après l'Arabie saoudite et l'Iran (CIA, 2006).

Ces dernières années, du fait de l'épuisement graduel des réserves pétrolières, le gouvernement a cherché à diversifier ses sources de revenus et à diminuer sa dépendance à l'égard du secteur énergétique. Cette diversification se caractérise notamment par le développement de l'industrie touristique, centrée sur les côtes, le désert, ou encore les gigantesques complexes sportifs (Dobel, 2005).
Son succès touristique - étroitement lié à d'autres facteurs comme les prix modérés des biens de consommation, une température élevée durant la majeure partie de l'année, mais aussi des projets d'infrastructure pharaonique comme le Burj Al-Arab, le Burj Dubai et The Palm Islands - a amené cette région à se désigner comme la « Singapour » ou la "Hong Kong du Moyen-Orient » (TNI, 2005). En 2003, les EAU ont accueilli 5,9 millions de touristes et ils ont bien l'intention de tripler ce chiffre d'ici 2010 (AME Info, 2005b).

Dubaï, l'un des sept émirats constituant les EAU, est également le nom de sa ville principale, s'il y a besoin de les différencier. La ville attire les touristes et les voyageurs d'affaires avec ses plages et ses autres attractions, comme l'immense hôtel Burj al-Arab qui a la forme d'une voile. La ville tente cependant, malgré les développements modernes, de maintenir un caractère arabe.

Selon les données du Dubai Department of Tourism and Commerce Marketing (DTCM), la ville a accueilli pas moins de 4,9 millions de touristes en 2003 (contre 4,7 millions en 2002 et 3,6 millions en 2001). L'année 2003 avait pourtant été une année plutôt moyenne en raison de la guerre en Irak et du SRAS (syndrome respiratoire aigu grave).
Un sondage, réalisé par la chaîne CNN au printemps 2004, classait la ville de Dubaï au troisième rang des meilleures destinations mondiales auprès des voyageurs d'affaires, derrière Hong Kong et Singapour. Pas étonnant alors qu'à l'été 2004, malgré la chaleur extrême (50 degrés Celsius), le taux d'occupation des hôtels y dépassait $85 \%$.

En ce qui concerne le secteur du voyage d'affaires, un sondage relayé par l'Association of Corporate Travel Executives (ACTE) affirmait que ce secteur devrait enregistrer une croissance de $5,5 \%$ dans les EAU cette année (comparé à 5,7 \% en 2004).

\section{L'Arabie saoudite}

L'Arabie saoudite est le principal pays de la péninsule arabique. Son économie repose principalement sur son industrie pétrolière. Depuis l'an 2000, elle connaît une forte amélioration de sa situation économique et financière grâce à l'augmentation des prix du pétrole et des quantités qu'elle met sur le marché mondial (de la Forest Divonne, 2005).

Les conditions d'accès à ce pays ne sont pas des plus faciles. En effet, un passeport valide y est exigé, tandis qu'il n'existe aucun visa de touriste. II n'est possible de s'y rendre pour affaires qu'à condition d'être invité par une entreprise ou un organisme local, ce qui permet d'obtenir un visa spécial. Le tourisme international s'y décline sous trois formes : le tourisme religieux (chaque année, plus de trois millions de pèlerins visitent le pays), les voyages d'affaires et la visite de parents et d'amis.

Cependant, dans sa politique d'ouverture aux investissements étrangers, l'Arabie saoudite a décidé de s'ouvrir peu à peu au tourisme d'agrément. C'est sous cette nouvelle impulsion qu'elle a créé récemment une commission de tourisme et que les autorités travaillent à définir de nouvelles politiques touristiques.

Depuis 1997 et en dehors du tourisme religieux, seuls quelques milliers de touristes sont venus découvrir les sites nabatéens du nord de la péninsule ou pratiquer la plongée sous-marine dans les eaux de la mer Rouge. 


\section{L'Égypte}

Pays chargé d'histoire et mondialement connu pour ses richesses archéologiques s'il en est, l'Égypte est en proie à de grandes difficultés économiques depuis plusieurs années. Avec une économie autrefois essentiellement agraire, elle tente maintenant de se diversifier en jouant la carte du tourisme.

Seulement, depuis le début des années 1990, son industrie du tourisme est bousculée par la recrudescence d'attentats terroristes islamistes (OMT, 2005). Citons, par exemple, une dizaine d'attentats contre les touristes qui ont lieu en Égypte de 1992 à 1997, année qui fut également marquée par deux attentats majeurs : en septembre, dix personnes (neuf Allemands et un Égyptien) ont été tuées dans un attentat perpétré contre un autocar de touristes devant le musée du Caire ; en novembre, 62 personnes (dont 58 touristes pour la plupart suisses et japonais) ont été tuées à Louxor. En octobre 2004 un triple attentat à la voiture piégée a été commis à Taba et à Ras Chetan dans le Sinaï contre des touristes israéliens (29 morts). Enfin, en 2005, il y a eu deux attentats majeurs, soit le 7 avril (attentat suicide dans les souks au Caire causant la mort de 3 touristes) et le 23 juillet (attentats à Charm el-Cheik).

Tous ces attentats semblent cependant n'avoir affecté le pays que durant de brèves périodes puisque, en 2004, le pays a comptabilisé plus de 8,1 millions de visiteurs, soit un taux de croissance de $11 \%$ par comparaison à 2003. La venue de ces visiteurs a rapporté pas moins de 7 milliards de dollars (contre 5,75 millions en 2003, un volume alors tout à fait comparable à celui de la Tunisie ou du Maroc).

Les quatre premiers mois de 2005 font apparaître une croissance encore supérieure, soit une variation de 15,6 \% par rapport à la même période de l'année précédente. Au Moyen-Orient, pendant la même période de l'année précédente, le nombre d'arrivées a presque triplé, pour s'établir à 35,6 millions en 2004. À l'échelle régionale, l'Égypte s'est taillé une part importante du marché, part qui, au cours de la décennie écoulée, est d'environ $23 \%$. Selon les estimations du ministère égyptien du Tourisme, le nombre de touristes étrangers en Égypte pourrait, en 2005, s'élever à 8,4 millions (Menara, 2005).

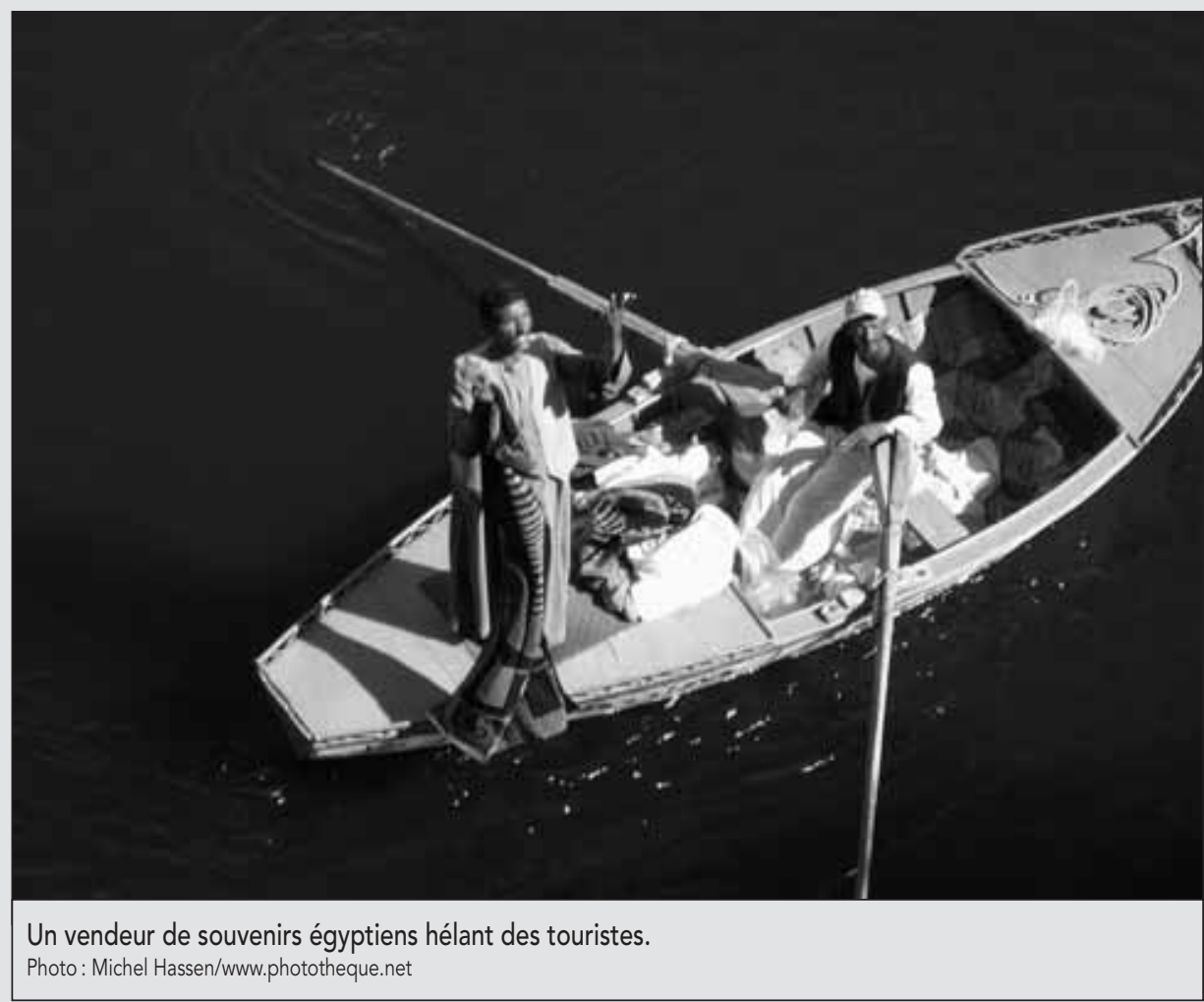

\section{La Jordanie}

L'économie de la Jordanie est fragilisée par deux grands problèmes: la pauvreté et le chômage. Cependant, en 2004, elle s'est particulièrement bien comportée, avec une croissance du PNB (produit national brut) de $7,4 \%$. Encouragées, les autorités tablent, si tout se passe comme prévu, sur une croissance de $6 \%$ du PIB en 2005. L'industrie, et encore plus la construction, connaissent un essor marqué (+20\% pour cette dernière) (EIU, 2005).

Les événements terroristes et les instabilités politiques qui ont cours actuellement en Palestine, en Irak et au Liban n'ont guère favorisé son développement touristique. Cependant, la Jordanie a vu, en 2004, une augmentation de $7,1 \%$ de ses visiteurs par rapport à 2003 (soit quelque 1,8 million de visiteurs en 2004, qui ont généré quelque 590 millions USD) (Shaqour, 2005).

Le secteur du tourisme représente l'une des quatre premières ressources du pays (avec les rapatriements de capitaux des expatriés jordaniens, l'aide internationale et le secteur minier). En 2004, il a généré quelque 864 millions USD de recettes (soit 7,5 \% du PIB) et employé 24000 personnes.
Le produit touristique y est très diversifié : le tourisme culturel (sites archéologiques), le tourisme sportif (plongée sous-marine), l'écotourisme et la découverte de la biodiversité (comme dans la vallée du Jourdain), le tourisme religieux (mont Nebo, site du baptême du Christ), le tourisme de santé (soit des cures dans les sources thermales de Maïn et d'El Hemma, les centres de thalassothérapie), le tourisme médical, etc.

\section{Le Yémen}

Bien que sa situation économique et financière soit fragile, ce pays n'inspire aucune inquiétude majeure à court terme. Le secteur des hydrocarbures y représente environ un tiers du PIB, $71 \%$ des recettes budgétaires et $92 \%$ des exportations. Son économie, la plus pauvre du golfe, repose essentiellement sur trois piliers : le pétrole, l'agriculture et les transferts des travailleurs émigrés (Yemen Observer Newspaper, 2005).

Dans ces conditions délicates, le Yémen fait systématiquement appel à l'aide internationale, soit sous la forme de rééchelonnements et d'annulations de dettes, et la dépendance à l'égard de l'aide internationale concerne surtout l'accès aux services de base comme l'eau, la santé, l'éducation et les transports. 
Le tourisme, plutôt embryonnaire, y est synonyme de culture, de patrimoine et de traditions. Le Yémen n'offre pas de perspectives en matière de tourisme de masse, bien que l'on constate que le nombre de touristes (principalement des Allemands, des Français, des Italiens et des Japonais) a augmenté depuis les quinze dernières années (Chaise, 2005).

À l'instar de Dubaï, le Yémen désire viser un tourisme sélectif, soit le tourisme de luxe. Le gouvernement yéménite espère pouvoir accueillir plus de un demi-million de touristes européens d'ici 2008 et ce, moyennant quelques améliorations aux infrastructures. Selon bon nombre de spécialistes du secteur, ce chiffre semble cependant exagéré, compte tenu que le réseau hôtelier est nettement insuffisant et que le pays est mal desservi sur le plan aérien (Yemen Observer Newspaper, 2005).

Actuellement, les agences de voyages proposent surtout des raids dans le désert, des visites culturelles et architecturales, de la plongée sous-marine, etc. La côte de la mer Rouge propose des plaisirs balnéaires, bien que celle-ci soit encore très peu aménagée. Mais c'est sans doute la sécurité qui représente le frein majeur au développement du tourisme dans cette région. En effet, le pays est toujours en proie à de vives tensions entre les différentes tribus. Et dans le contexte irakien, il persiste au Yémen un fort sentiment anti-occidental.

Des pays comme la Syrie et le Liban font par ailleurs l'objet d'une analyse plus approfondie et ciblée dans ce numéro.

\section{Des investissements pharaoniques}

Selon une étude réalisée en 2006 par la firme Accenture, et malgré toutes les données conjoncturelles citées plus haut, l'industrie du tourisme et du voyage au Moyen-Orient devrait générer pas moins de 147,6 milliards USD en 2006 et 279,4 milliards USD en 2016 (WTTC, Accenture, 2006). Les analystes du World Travel \& Tourism Council (WTTC) prévoient une croissance de $4 \%$ en 2006 et de 4,4\% par année de 2007 à 2016 (WTTC et Accenture, 2006). Les secteurs du tourisme et du voyage devraient représenter, selon eux, 2,6 \% du PIB du Moyen-Orient en 2006 (soit 27,3 billions USD), croissant ensuite jusqu'à 3,1\% en 2016 (soit l'équivalent de 58,9 billions USD).
En nombre d'emplois générés, cela donne une estimation de 4590000 emplois en 2006 (soit $10,1 \%$ de la masse salariale totale), passant à 6141000 emplois en 2016 (soit 10,6\% de la masse salariale totale).

Tous conscients de la nécessité de devoir développer le secteur du tourisme afin de pallier une baisse de production du pétrole à plus ou moins long terme et pour diversifier une économie souvent basée sur une seule économie, les pays du Moyen-Orient ont décidé d'investir massivement dans le développement et la promotion touristique, chacun à sa manière et à son rythme. Certains pays choisissent d'investir dans la construction de projets majeurs (hôtels, aéroports, ports de plaisance, etc.), tandis que d'autres dévoilent d'ambitieux programmes de développement touristique. Voyons brièvement comment ces investissements se déclinent dans quelques-uns de ces pays.

L'Égypte a choisi d'encourager fortement les investissements privés. Sur la côte méditerranéenne, dans le nord du pays, de nombreux projets touristiques majeurs (Taba, Marsa Alam, Alamein) ont été développés par le secteur privé. Les ports de la mer Rouge, de Hurghada et de Safaga ont été agrandis afin de mieux servir l'industrie de tourisme et de pouvoir accueillir des bateaux de grande capacité. On parle également de projets majeurs d'expansion dans les aéroports du pays, dans les chemins de fer, ainsi que du développement du métro au centre-ville du Caire.

C'est en mai 2005 que le gouvernement du Qatar dévoilait son nouveau programme touristique, soit un investissement de 15 milliards USD dans des hôtels de luxe, des projets culturels, des équipements sportifs (golf, tennis...) pouvant accueillir des événements internationaux (WTTC, 2004). Pour recevoir les voyageurs internationaux, le programme inclut également le développement d'un nouvel aéroport international (au coût de 5 milliards USD) à Doha, la capitale. Cet aéroport de dernier cri devrait ouvrir ses portes en 2008.

En Syrie aussi, l'inéluctable développement est en marche. Le gouvernement a énergiquement investi dans la modernisation des infrastructures (routes, réseaux hydrauliques, télécommunications, hôtellerie, mise en valeur du patrimoine bâti, etc.). À la contribution de l'État se sont ajoutés des investissements privés. Des promoteurs privés étrangers ont investi en 2004 un peu plus de 3 milliards USD. De nombreux projets de villages de vacances et de complexes hôteliers sont en route (Dayah, 2005). Mais le plus impressionnant développement vient sans aucun doute de la part de Dubaï. Ville au superlatif, Dubaï se revendique comme étant LE haut lieu du tourisme et des affaires dans le golfe Persique (Belkaïd, 2004). Les hautes tours et les hôtels y rivalisent de luxe, d'élégance et d'extravagance. Et tandis que l'annonce de nouveaux projets immobiliers s'y fait à coups de milliards de dollars, le gouvernement met sur pied une politique d'attraction concrétisée par des situations fiscales plus qu'intéressantes, ainsi que par la mise en place d'événements importants et la construction de projets touristiques, hôteliers et immobiliers pharaoniques (Laliberté, 2004).

Pour 2010, Dubaï ne cible pas moins de 15 millions de visiteurs, ce qui nécessite une consolidation dans les transports aériens afin de rendre ceux-ci plus accessibles, ainsi qu'une augmentation du secteur hôtelier de classe moyenne. Certains analystes prévoient que, d'ici là, l'industrie du tourisme contribuera pour $20 \%$ du PIB national, soit une augmentation de $8 \%$ par rapport à 2004. En 2004, la répartition des touristes s'élevait à $40 \%$ de touristes d'affaires contre $60 \%$ d'agrément. D'ici 2010, Dubaï espère augmenter la portion des touristes venant pour les loisirs grâce à Dubailand, un gigantesque complexe de loisirs actuellement en développement (AME Info, 2005b).

\section{Image de marque et stratégies de promotion}

L'image du Moyen-Orient est encore bien souvent associée aux conflits politiques, à l'extrémisme et aux attentats terroristes (Easen, 2006). Pour pallier cette image parfois négative qu'a le touriste nonarabe, chaque pays rivalise de stratégies promotionnelles.

Au Qatar, par exemple, on tente de jouer la carte de la culture (Guez, 2004). En effet, afin de contrer l'arrivée du tourisme de masse qui envahit Dubaï, ce pays a décidé de se lancer dans une ambitieuse politique culturelle pour en faire une destination « intellectuelle». II s'est donc lancé dans plu- 
sieurs projets d'envergure tels que : la création de pôles universitaires, la construction d'une grande bibliothèque (qui contiendra également un musée, des galeries d'art, des salles de conférence, des restaurants et des cafétérias), la promotion de sites historiques, la création d'un musée des arts islamiques (45000 mètres carrés comprenant une bibliothèque, des galeries, des jardins et un restaurant - ouverture prévue en 2006), la reconstruction du musée national du Qatar et, enfin, la construction d'un nouveau musée de la photographie (11000 mètres carrés de galeries de photographies, d'expositions d'appareils photo, des ateliers, des salles de conférences, des restaurants et des cafétérias - ouverture prévue en 2007), etc.

Désormais, le pays se veut une destination touristique de haute qualité pour le tourisme culturel et d'affaires, cherchant à mettre en valeur ses plages, ses hôtels prestigieux, ses centres commerciaux. Pour 2010, le Qatar entend bien attirer 1,4 million de visiteurs (contre 500000 en 2004), en développant le tourisme d'affaires, sportif et médical, ainsi que les conférences.

Au Sultana d'Oman, on a plutôt choisi la carte de la promotion par le bouche-à-oreille. Déjà, en 1999, plus de 350 tour-opérateurs et journalistes y avaient été invités afin de juger par eux-mêmes de la potentialité touristique du pays. Depuis lors, de nombreux opérateurs étrangers viennent chaque année et les congrès et les colloques internationaux n'ont pas cessé de se multiplier (Choufany et Younes, 2005). De plus, plusieurs offices du tourisme omanais ont été ouverts dans des grandes villes telles que Londres, New York, Hong Kong, Berlin, Madrid, Genève et Paris afin d'en faire la promotion.

Oman est un pays à la croisée des chemins qui s'ouvre peu à peu au monde. II a, jusqu'à récemment, peu misé sur le tourisme qui est au stade du développement depuis quelques années. En effet, ce n'est qu'en juin 2004 qu'il s'est doté d'un ministère du Tourisme. Conscient de la nécessité d'investir dans ce nouveau créneau, le gouvernement a décidé d'augmenter le budget marketing du tourisme de 10 millions USD en 2004 à 30 millions en 2005. Un autre 220 millions est déjà budgété pour promouvoir le tourisme au cours des cinq prochaines années (Brasselet, 2005).
Étant donné le potentiel significatif du marché et l'engagement du gouvernement à accroître les investissements dans cette industrie, on s'attend à ce que le sultanat connaisse une forte croissance. D'ici 2010, environ 5 milliards USD devraient y être investis (Choufany et Younes, 2005).

Déjà, afin de favoriser et de faciliter l'entrée des touristes, le gouvernement a entamé des démarches dans le but d'alléger les procédures d'obtention de visas, permettant ainsi aux visiteurs de 68 pays de se procurer un visa dès leur arrivée à l'aéroport ou au passage des frontières.

Au Moyen-Orient, un pays a décidé de promouvoir le tourisme en grandes pompes. C'est le cas de la Jordanie, où le ministère du Tourisme (MoTA) a inauguré officiellement, en septembre 2004, la Journée internationale du tourisme, ainsi que la nouvelle stratégie nationale du tourisme (SNT) 2004-2010. Les quatre principaux objectifs de cette stratégie sont :

- d'attirer 12 millions de touristes d'ici l'an 2010 ;

- d'augmenter les recettes touristiques à hauteur de 1,8 milliard USD ;

- d'augmenter les recettes fiscales d'un montant de 640 millions USD ;

- et, enfin, de créer 51000 nouveaux emplois et des milliers de PME.

Pour atteindre ses objectifs, la Jordanie prévoit développer et renforcer sa mise en marché (en allouant par exemple $2 \%$ des budgets de 2005 et de 2006 au marketing) ; développer et diversifier le produit touristique; développer les ressources humaines; instaurer une réforme juridique et institutionnelle; et, enfin, favoriser les investissements étrangers et le partenariat public/privé (PPP). En raison de ces nombreux avantages, les dernières années ont vu de nombreux et d'importants investissements dans l'industrie touristique en général et dans le secteur hôtelier en particulier. D'Aqaba à la mer Morte, des projets de développement gigantesque sont soit à l'étude, soit en cours de réalisation.

Quant à la Syrie, elle tente depuis quelques années de se tailler une place parmi les géants du tourisme mondial en ciblant les clientèles potentielles des pays d'Asie, d'Europe et d'Afrique du Nord (Grim, 2005). Parallèlement aux développement et à la mo- dernisation des infrastructures immobilières et hôtelières, le gouvernement syrien a bien l'intention de tout faire pour ériger le tourisme en tant que moteur du développement économique et social. C'est donc à grands coups de publicité et d'actions marketing importantes qu'il promeut les produits touristiques régionaux comme: le Festival de la route de la soie, les sites archéologiques, les hauts lieux de la culture et de l'histoire empreints de traditions culturelles, religieuses, artisanales, culinaires, etc.

D'ici 2010, le ministère du Tourisme propose trois défis qui sonnent déjà comme une devise : "promotion, qualité et compétitivité ". Les principaux objectifs du ministère sont d'atteindre une moyenne de 10000 lits/an et des investissements de un milliard USD/an ; 8 millions de touristes en 2010, avec des recettes de 6 milliards USD ; et de nouveaux produits à prix modéré.

Un autre pays met également les bouchées doubles: Israël, qui, après des années de recul dues à la situation politique régionale, a décidé de faire « redécoller » son industrie du tourisme, lourdement hypothéquée sur le plan de la confiance des voyageurs. Le ministère du Tourisme multiplie les initiatives afin de relancer le tourisme israélien qui reprend doucement du mieux après avoir été sinistré par cinq années d'Intifada (FWW OnLine, 2005). Soucieux de redorer son image de marque, il a décidé de promouvoir des projets touristiques, de concert avec l'Égypte, la Jordanie et l'autorité palestinienne. La concertation des opérateurs touristiques ira de pair avec la coordination sécuritaire et la facilitation des conditions respectives d'octroi de visas (Agence France Presse, 2005).

Tel que décrit ci-dessus, I'Arabie saoudite n'est guère ouverte au tourisme de masse. Plus timidement, le pays souhaite néanmoins assouplir ses règles et distribue depuis peu des brochures touristiques où le slogan définit l'essence même de son histoire: "L'Arabie saoudite, le pays inconnu». Pour appuyer ce message, le royaume participe depuis peu aux grands salons internationaux du tourisme.

Comme autre forme de promotion et de mise en valeur, certains pays choisissent de mettre l'accent sur un événement mondial qui leur procurera une vitrine incroyable sur le tourisme. C'est le cas du Grand Prix du 
Bahrein, une épreuve comptant pour le championnat du monde de Formule 1. Créé en 2004 sur le circuit international de Bahrein et commandité par Gulf Air, cette course entre dans I'histoire comme étant le premier Grand Prix de Formule 1 à se dérouler au Moyen-Orient.

Enfin, le lancement récent d'un canal de nouvelles de télévision Al-Jazeera anglophone devrait permettre à certaines destinations moyen-orientales de proposer une fenêtre sur de nouveaux segments de la culture arabe, de briser les tabous en remplaçant le tout par des slogans davantage à l'image de la région. Cette nouvelle chaîne devrait assurément aider à rétablir les images tant du tourisme que de la culture locale (Easen, 2006).

\section{Conclusion}

Le Moyen-Orient est un ensemble de pays qui bénéficient d'un environnement économique favorable au développement de l'industrie touristique et tout indique un fort potentiel d'investissements dans l'avenir. Ces développements sont, bien sûr, toujours tributaires de la situation politique qui constitue la principale fragilité de la région.

Afin de favoriser leur avantage concurrentiel, ces destinations se présentent au monde sous leur meilleur jour et les splendeurs du monde arabe se déclinent maintenant sous diverses formes, que ce soit le trekking dans les montagnes de Haraz au Yémen, la plongée sous-marine dans la mer Rouge ou même le ski dans le désert. À coups de millions, elles investissent massivement, à la fois dans l'amélioration des infrastructures (que celles-ci soient hôtelières, immobilières, de transport, etc.), ainsi que dans la publicité et la promotion en général.

Le temps est venu de rassurer les touristes et de gagner leur confiance. Rien n'est trop beau ni trop grand, le Moyen-Orient est bien décidé à tourner la page sur les instabilités politiques.

Françoise Mommens est chargée de veille au Réseau de veille en tourisme (Chaire de Tourisme, ESG-UQAM).

\section{Bibliographie}

Agence France Presse (2005), Tourisme : Israël, l'Égypte, la Jordanie et les Palestiniens coopèrent, 14 septembre, [n'est plus en ligne].

AME Info (2005a), The Middle East Tourism Paradox, [http://www.ameinfo.com/39057 .html], mai.

AME Info (2005b), Tourism Profile of Dubai to Shift by 2010, [http://www.ameinfo.com/67872. html], 19 septembre.

Belkaïd, Akram (2004), " Les ambitions de Dubaï, 'Hong Kong' du golfe Persique », La Tribune, Desfossés, 14 juin.

Brasselet, Nadine (2005), Le tourisme en Oman, Étude de la mission économique MINEFI/ DGTPE, Ambassade de France en Oman, Mission économique de Mascate, fiche de synthèse, 7 juin.

Chaise, Christian (2005), " Les Occidentaux commencent à revenir au Yémen », Le Soleil, 26 mars.

Choufany, Hala Matar, et Elie Younes (2005), "Oman - Today's Spotlight, Tomorrow's Destination ", HVS International, [http://www.hvsin ternational.com/Content/1687.pdf], juin.

CIA (2006), The World Factbook, [http://www. cia.gov/cia/publications/factbook].

Dayah, Nida (2005), Le secteur du tourisme en Syrie, Étude de la mission économique MlNEFI/DGTPE, Ambassade de France en Syrie, fiche de synthèse, 18 septembre.

de la Forest Divonne, Bertrand (2005), Arabie saoudite, Étude de la mission économique MlNEFI/DGTPE, Ambassade de France en Arabie saoudite, fiche signalétique, 17 mars.

Dobel, Christiane (2005), Le secteur du tourisme aux Émirats arabes unis, Étude de la mission économique MINEFI/DGTPE, Ambassade de France aux Émirats arabes unis, fiche de synthèse, actualisation janvier.

Easen, Nick (2006), « Middle East Turns to Positive Images ", Hotel Travel News, 10 mai.

FW OnLine (2005), Israel Seeks Return to Tourism Map, [http://www.fvw.com], 21 septembre.

Grim, Nordine (2005), « La Syrie veut figurer parmi les grands du tourisme mondial ", El Watan, 9 octobre.

Guez, Olivier (2004), « Le Qatar, un laboratoire du renouveau arabe ", La Tribune (France), 14 juin.

Gyrard, Denys (2005), Hôtellerie et restauration à Bahreïn, Étude de la mission économique MINEFI/DGTPE, Ambassade de France à Bahreïn, fiche de synthèse, 20 juillet.

Heyer, Hazel (2005), « Lebanon in a Transition, Says Tourism Minister », Trave/WireNews, 30 septembre.

Laliberté, Michèle (2004), « Dubaï, région de la démesure ", Réseau de veille en tourisme, [http://www.veilletourisme.ca/fr/titre_resu me_article.aspx?sortcode=1.5.17\&id_article =311], 3 novembre.

Menara (2005), L'Égypte prévoit 8,4 millions de touristes en 2005, 2 décembre.

Organisation mondiale du tourisme (OMT) (2005a), Baromètre OMT du tourisme mondial, [http:// www.world-tourism.org/facts/eng/pdf/baro meter/WTO_Barom05_2_excp_fr.pdf], juin.

Organisation mondiale du tourisme (OMT) (2005b), Le tourisme en Égypte et l'attentat de Sharm el-Sheikh, Premier rapport spécial sur l'impact de l'attentat de Sharm el-Sheikh, 11 août.

Shaqour, Ghada (2005), Secteur du tourisme en Jordanie, Étude de la mission économique MINEFI/DGTPE, Ambassade de France en Jordanie, fiche de synthèse, octobre.

The Economist Intelligence Unit (EIU) (2005), " Middle East and Africa: Travel and Tourism at-a-Glance ", Travel and Tourism Forecast Middle East and Africa, juin.

Tourisme News International (TNI) (2005), Voyage d'affaires : les Émirats arabes unis enregistrent une croissance régulière, [http://www. tnitourisme.com], 16 novembre.

World Travel \& Tourism Council (WTTC) (2004), Qatar Unveils New Tourism Masterplan to Become a World-Leading High-Quality Tourist Destination, 1er mai, [http://www. wttc.org/news63.htm].

World Travel \& Tourism Council (WTTC) et Accenture (2006), Middle-East. Travel and Tourism Climbing to New Economic Research: The 2006 Travel and Tourism Economic Research, [http://www.wttc.org/2006TSA/ pdf/World.pdf], 24 février.

Yemen Observer Newspaper (2005), «A Flourishing Year for Tourism in Yemen ", écrit par l'équipe de rédaction, vol. VIII n 47, 3 décembre.

Younes, Elie, et Bernard Forster (2005), « Middle East Hotels Market: Outlook, Trends and Opportunities ", HVS International, [http://www. hvsinternational.com/Content/1630.pdf]. 